\section{Health-related quality of life in IBS}

Despite guidelines recommending its routine evaluation, health-related quality of life (HRQOL) tends to be measured inaccurately in patients with irritable bowel syndrome (IBS). To help clinicians to provide better assessments, Spiegel et al. have identified a set of clinical determinants of HRQOL in patients with this condition.

The team selected 30 hypothesis-driven predictors of HRQOL, covering demographics, bowel symptoms, psychological symptoms and use of health care resources. Each of these was expressed as a dichotomous variable to simplify interpretation. A total of 770 patients with IBS were then examined using a symptom questionnaire. All patients also completed the 36-Item Short-Form Health Survey (SF-36), which is a validated, generic HRQOL instrument. Global HRQOL, as indicated by the SF-36, was the main outcome of the study.

Multivariate regression analysis showed that seven of the hypothesis-driven variables independently predicted physical HRQOL, whereas eight factors were similarly associated with mental HRQOL. Two variables ('tiring easily' and 'low in energy') were common to both the physical and mental HRQOL domains. Physical HRQOL was largely associated with the severity, periodicity and pain of symptoms. Mental HRQOL, however, was predicted by problems with sexual function/interest, mood and anxiety.

Concluding that overall HRQOL was mainly associated with extraintestinal symptoms, the authors recommend that factors such as stress and anxiety should be addressed. They note that the clinical determinants identified in this study might prove useful in assessing HRQOL in IBS.

Original article Spiegel BMR et al. (2004) Clinical determinants of health-related quality of life in patients with irritable bowel syndrome. Arch Intern Med 164: 1773-1780

\section{Live donor liver transplantation: graft and patient survival}

In response to an acute cadaver organ shortage, live donor liver transplantation (LDLT) is becoming more common. The procedure is associated with significant donor morbidity and a degree of donor mortality. It is important, therefore, to balance the risk to the donor against the likelihood of a positive outcome in the recipient. To this end, Thuluvath and Yoo have assessed the outcomes in patients who underwent LDLT, compared with those subjected to deceased donor liver transplantation (DDLT).

Using data from the United Network for Organ Sharing database, the authors identified all adults who underwent LDLT in the US between 1988 and 2001. Most of the 764 procedures had been performed since 1998. Case-controls $(n=1,470)$, who underwent DDLT during the same period, allowed comparison of rates of graft and patient survival.

Primary graft nonfunction and acute rejection occurred at similar rates in patients who underwent LDLT or DDLT. Patient survival at 2 years was also similar in the two groups (79.0\% in LDLT vs $80.7 \%$ in DDLT; $P=0.5$ ). Two-year graft survival, however, was significantly lower in the LDLT group compared with the DDLT group $(64.4 \%$ vs $73.3 \%, P<0.001)$. This corresponded to a $60 \%$ higher risk of graft loss in the LDLT group, after adjusting for confounding variables.

Commenting that outcomes can be expected to improve as experience of LDLT is accumulated, Thuluvath and Yoo conclude that the procedure is a reasonable option in cases where DDLT is not available.

Original article Thuluvath PJ and Yoo HY (2004) Graft and patient survival after adult live donor liver transplantation compared to a matched cohort who received a deceased donor transplantation. Liver Transpl 10: 1263-1268

\section{Extrahepatic bile duct carcinoma without jaundice}

Jaundice is the initial sign of extrahepatic bile duct carcinoma (EBDC) in the majority of patients, and is an important factor in the diagnosis of the disease. It is possible, therefore, that EBDC patients without jaundice may be underdiagnosed or misdiagnosed. Tang and colleagues have explored this in their recent study.

A total of 101 patients were treated surgically for EBDC at a single center in China, between 1985 and 1999. On the basis of preoperative serum total bilirubin levels, 84 of the patients had jaundice and 17 did not. The 